\title{
Advances in Immunotherapy for Glioblastoma Multiforme
}

\author{
Boyuan Huang, ${ }^{1}$ Hongbo Zhang, ${ }^{2}$ Lijuan Gu, ${ }^{3}$ Bainxin Ye, ${ }^{4}$ Zhihong Jian, ${ }^{5}$ \\ Creed Stary, ${ }^{6}$ and Xiaoxing Xiong ${ }^{3,5}$ \\ ${ }^{1}$ Department of Neurosurgery, Beijing Electric Hospital, State Grid, Beijing 100073, China \\ ${ }^{2}$ Department of Neurosurgery, Hubei Provincial Hospital of Integrated Chinese and Western Medicine, \\ Wuhan, Hubei 430015, China \\ ${ }^{3}$ Central Laboratory, Renmin Hospital of Wuhan University, Wuhan, Hubei 430060, China \\ ${ }^{4}$ Department of Hematology, Renmin Hospital of Wuhan University, Wuhan, Hubei 430060, China \\ ${ }^{5}$ Department of Neurosurgery, Renmin Hospital of Wuhan University, Wuhan, Hubei 430060, China \\ ${ }^{6}$ Department of Anesthesiology, Perioperative and Pain Medicine, Stanford University School of Medicine, \\ Stanford, CA 94305, USA
}

Correspondence should be addressed to Creed Stary; cstary@stanford.edu and Xiaoxing Xiong; xiaoxingxiong@whu.edu.cn

Received 15 June 2016; Revised 15 January 2017; Accepted 26 January 2017; Published 19 February 2017

Academic Editor: Menaka C. Thounaojam

Copyright (c) 2017 Boyuan Huang et al. This is an open access article distributed under the Creative Commons Attribution License, which permits unrestricted use, distribution, and reproduction in any medium, provided the original work is properly cited.

\begin{abstract}
Glioblastoma multiforme (GBM) is the most common primary malignant brain tumor in adults. Patients with GBM have poor outcomes, even with the current gold-standard first-line treatment: maximal safe resection combined with radiotherapy and temozolomide chemotherapy. Accumulating evidence suggests that advances in antigen-specific cancer vaccines and immune checkpoint blockade in other advanced tumors may provide an appealing promise for immunotherapy in glioma. The future of therapy for GBM will likely incorporate a combinatorial, personalized approach, including current conventional treatments, active immunotherapeutics, plus agents targeting immunosuppressive checkpoints.
\end{abstract}

\section{Introduction}

Glioblastoma multiforme (GBM) is the most common primary malignant brain tumor in adults, accounting for approximately $60-70 \%$ of gliomas [1] and $15 \%$ of primary brain tumors [2]. The current standard treatment for patients with GBM is maximal tumor resection followed by adjuvant radiotherapy and temozolomide [3]. Although this standardized treatment has demonstrated efficacy in prolonging patient survival, the prognosis for patients remains extremely poor, with a median survival time (MS) of 14.6 months and an average 5 -year survival rate of less than $5 \%[1,2,4]$. This may be partly due to resistance of GBM cells to treatment and their capacity to spread and invade into surrounding brain parenchyma. Accordingly, substantial efforts have been made in developing new approaches for gene therapy, targeted chemotherapeutics, and/or radiotherapeutic modalities. However, the MS for patients with newly diagnosed GBM have improved only modestly during the past 10 years.
Immunotherapy, harnessing the power of the host's immune system by inducing, enhancing, or suppressing immune responses to reject cancer cells, is rapidly becoming a pillar of anticancer therapy. Immunotherapeutic approaches can be classified as active immunotherapy aimed at promoting a $\mathrm{T}_{\mathrm{h}} 1$ immune response through tumor vaccines, nonspecific immune stimulants, or cellular vaccines, and passive immunotherapy, to induce an antitumor effect by transferring effector immune cells into patients. In 2010, the first antigen-specific vaccine for castration-resistant prostate cancer, sipuleucel-T, was approved by the FDA. In 2011, the first checkpoint inhibitor for advanced melanoma, ipilimumab, was also approved. Since then, immunotherapy has proven effective in the treatment of melanoma, Hodgkin's lymphoma, renal cell carcinoma, and non-small-cell lung cancer (NSCLC) in which conventional therapies have gained limited success [5-9] (Table 1). In this review, we will summarize the application of immunotherapy for GBM and discuss preclinical data and emerging clinical studies of vaccination, 
TABLE 1: Stage of clinical development of immunotherapeutics in select cancers.

\begin{tabular}{lccc}
\hline Cancer type & Mechanism & Agent & Phase \\
\hline Melanoma & Anti-CTLA-4 & Ipilimumab & FDA approved \\
Melanoma & Anti-PD-1 & Nivolumab & Phase III \\
Melanoma & Anti-PD-1 & Pembrolizumab & FDA approved \\
Melanoma & Adoptive cell therapy & Phases I-II \\
Melanoma & Peptide Vaccine & Melan-A peptides & Phases I-II \\
Melanoma & Autologous DC vaccine & & Phases I-II \\
Melanoma & Whole tumor cell vaccine & Phases I-II \\
\hline NSCLC & Peptide vaccine & Phase III \\
NSCLC & Anti-CTLA-4 & EGFR peptide & Phase III \\
NSCLC & Anti-PD-1 & Ipilimumab & Phase II \\
\hline
\end{tabular}

immune checkpoint blockade, and adoptive T-cell transfer in the treatment of this devastating disease.

\section{CNS Immune Privilege and Immunosuppression of GBM}

The central nervous system (CNS) has been traditionally viewed as an immune-privileged site, secondary to the bloodbrain barrier (BBB) that prevents free diffusion of cells and molecules and lack of a conventional lymphatic drainage system [10-13]. Paradoxically, however, it has been known for over 20 years that brain tumors have the capacity to elicit potent antitumor immune responses. Most recently, the discovery of a CNS lymphatic system has provided an explanation for this phenomenon. Using animal bearing intracranial tumors models, it has been demonstrated that tumor-derived antigens can be drained from the cerebrospinal fluid into the cervical lymph nodes to stimulate specific T-cells [14]. After amplification, these T-cells are able to efficiently migrate into the CNS and target and kill tumor cells [15]. However, these so-called tumor-specific T-cells have to exert their function in a hypoxic environment, where chronic inflammation and tumor cells can stimulate immunosuppression [16]. In addition, the inflammatory stimuli introduced by brain tumors can induce microglial activation and blood-brain barrier (BBB) disruption. Microglia serve as the main effector cells of the innate immune system in the CNS and play a critical role in cytotoxicity against phagocytosis and T-cell activation through antigen presentation. It has been demonstrated that microglia can increase GBM cell migration and invasion via secretion of matrix-degrading enzymes and membrane type I metalloproteinases (MMPs) [17]. The role that microglia plays in GBM tumor progression was verified by the identification of protumorigenic Osteoactivin (GPNMB) and Osteopontin (SP1) expression in profiled GBM tumor-associated microglia [18]. Disruption of the $\mathrm{BBB}$ with injury and disease can facilitate the presentation of CNS antigens to the cervical lymph nodes, serving to prime T-cells for homing and infiltration into the tumor parenchyma [19-21].

In GBM, a high level of vascular endothelial growth factor (VEGF) expression and pathologically structured microvessels can introduce increased permeability of BBB, enhancing the interaction between tumor cells and the immune system. GBM cells express high levels of MHC and Fas which play a role in the adaptive immune response. However, GBM has been traditionally considered an immunosuppressive tumor, effective in evading the immune response through a variety of mechanisms (Figure 1). First, GBM can express various potent immunosuppressive factors, such as indoleamine 2,3dioxygenase (IDO), TGF- $\beta$, and STAT3 [22-24]. IDO is expressed in $96 \%$ of resected GBM, of which the upregulation is correlating with a poor patient prognosis [25]. IDO1 functions to convert tryptophan into kynurenines, which mediate apoptosis of effector T-cells and activation of regulatory $\mathrm{T}$ cells- (Treg-) mediated immunosuppression [26]. Inhibition of TGF- $\beta /$ Smads signaling can restore immune surveillance in glioma models [27] which could inhibit proliferation through microRNA-182 and platelet-derived growth factor$\beta$ (PDGF- $\beta$ ). Second, another immunosuppressive pathway mediated by interactions between programmed death 1 (PD1) and programmed death-ligand 1 (PD-L1) contributes to the inhibition of T-cell activation and proliferation. Examination of 135 GBM specimens demonstrated that PD-L1 was positively expressed in $88 \%$ newly diagnosed GBM patients and $72 \%$ recurrent GBM patients [28]. Although the PDL1 expression in the healthy CNS parenchyma surrounding GBM is very low, GBM cells express a relatively higher level of PD-L1 than other tumors ( $30 \%$ of melanomas [29] and $25-36 \%$ of NSCLC [30]). Moreover, both tumor-infiltrating macrophages and microglia in GBM were reported to express high levels of PD-L1, suggesting the need for optimal immunotherapeutic benefit [31, 32]. A third predominant and essential pathway contributing to immunosuppression in GBM is mediation by cytotoxic T-lymphocyte antigen4 (CTLA-4), a coinhibitory receptor that outcompetes costimulatory receptor, CD28, for binding to CD80 and CD86 $[33,34]$. The inhibitory effects of CTLA-4 occur largely in naive and resting $\mathrm{T}$-cells and act to inhibit $\mathrm{T}$-cell effector function and augment the inhibitory activity of Tregs [35].

\section{T-Cell Based Vaccine Therapies}

Recent expansion in our knowledge of immune-mediated mechanisms has led to the rapid development of immunetargeted therapeutic strategies (Table 2). Among anticancer 


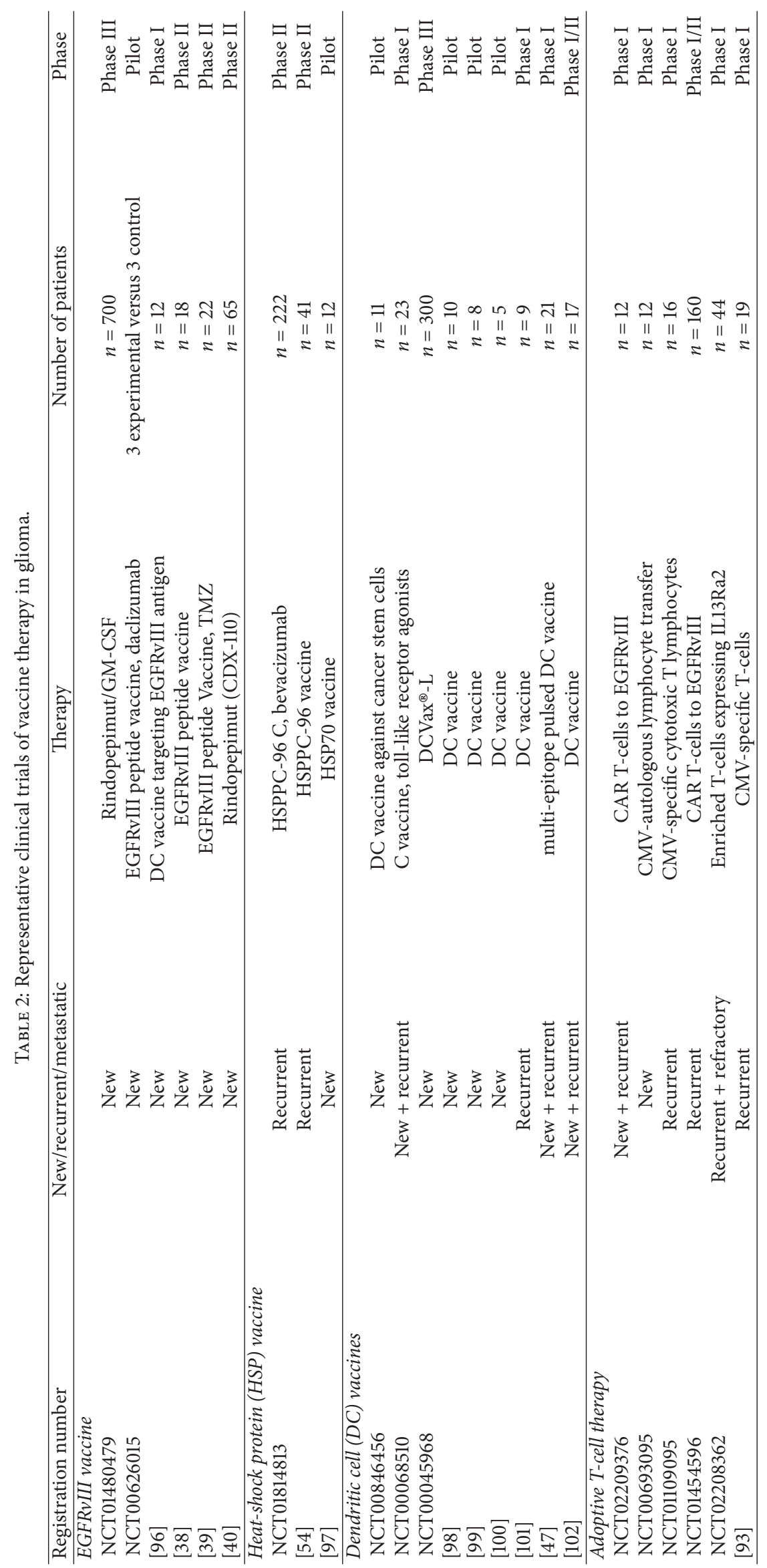



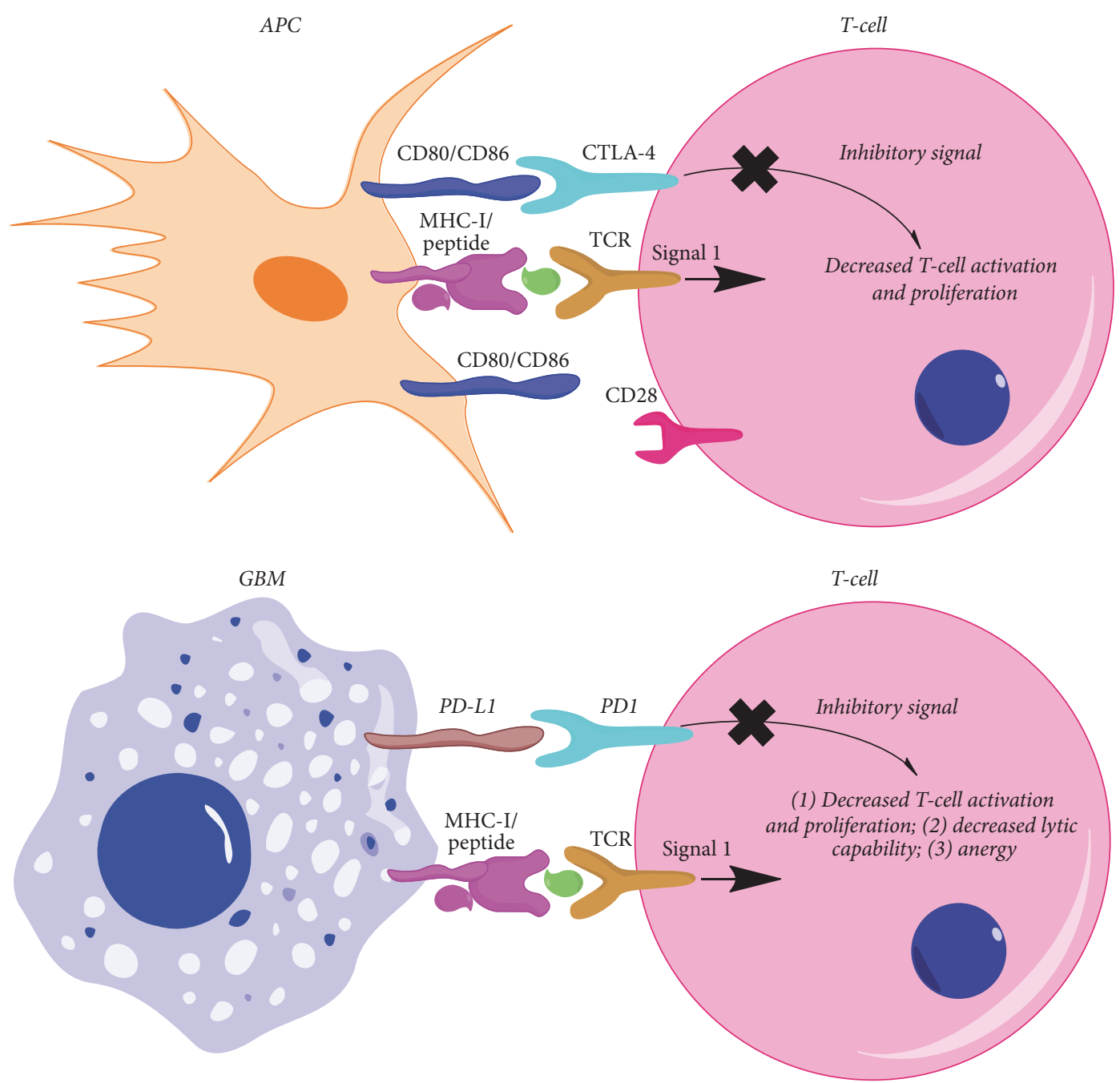

FIGURE 1: Mechanism of CTLA-4 and PD-L1 immune checkpoints. The CTLA-4 immune checkpoint (left figure) occurs early during the priming phase of the immune response, acting within secondary lymphoid organs. CTLA-4 is a powerful inhibitory T-cell receptor that can preferentially bind to CD80/CD86 on the surface of APCs, preventing their binding to the T-cell costimulatory receptor CD28, thus leading to decreased T-cell activation and proliferation in the context of antigen-presenting MHC class I. PD-1 signaling takes place during the effector phase of the immune responses within the tumor microenvironment. The inhibitory PD-1 T-cell receptor interacts with one of two currently identified PD-1 ligands: PD-L1 or PD-L2, expressed on the surface of tumor cells. Engagement of PD-1 ligands with the PD-1, in the context of tumor antigen-presenting MHC class I, can decrease the T-cell tumor lytic capacity and induces T-cell anergy. APC: antigen-presenting cell.

immunotherapies, the success of tumor vaccines and Tcell therapies relies on the elicitation of significant numbers of tumor-specific T-cells to seek and destroy tumor cells. Adaptation of vaccination strategies in cancer is aiming at eliciting unproductive immune responses against tumor cells in the patient by injection of tumor-derived antigens. The primary requirement for a safe and effective tumor vaccine is that the antigen target be expressed specifically in tumor cells but absent in normal cells of the body. In this regard, tumor-specific antigens (TSAs) that arise from mutations in the tumor are ideal candidates. An example of a potential TSA in GBM is epidermal growth factor receptor variant III (EGFRvIII), which induces the immune system to act against the tumor by presenting the mutant peptide to the stimulated immune cells [36]. EGFRvIII is the result of an in-frame deletion of exons 2-7 on EGFR resulting in a novel amino acid sequence and a truncated protein with an altered extracellular domain epitope [37]. Phase II clinical trials of Rindopepimut ${ }^{\mathrm{TM}}$, a 13-amino acid EGFRvIII peptide vaccine conjugated to adjuvant has demonstrated vaccine immunogenicity and increased overall survival (OS), which is correlated with the magnitude of induced tumor immunity [38-40]. Interestingly, most patients that relapsed after vaccination had lost the EGFRvIII antigen, demonstrating at the same time the efficacy of vaccine-induced immune responses in eradicating tumor cells [38]. Another phase III ACT IV study involved 700 patients with newly diagnosed EGFRvIII-positive GBM demonstrated that treatment of Rindopepimut (Rintega) plus temozolomide failed to improve overall survival (OS) compared with temozolomide 
and a control [41]. However, as reported in a study using single-cell DNA analysis, only a subset of cells in the tumor may express EGFRvIII due to the intratumoral heterogeneity, and expression may be highly variable $[42,43]$, resulting in survival and recurrence of the non-EGFRvIII-expressing cells. Despite these concerns, trials of Rindopepimut have shown promising results overall, leading to an ongoing phase III trial in newly diagnosed (NCT01480479) and relapsed (NCT01498328) GBM. Unfortunately, since EGFRvIII is only present in $20-30 \%$ of newly diagnosed GBM [44], the identification of alternative GBM TSAs with higher levels of expression will likely be necessary to achieve higher efficacy. For example, another clinical trial based on the mutant isocitrate dehydrogenase type 1 (IDH1) for recurrent grade II astrocytoma (NCT02193347) has shown greater efficacy [45]; the mutant IDH1 is carried by more than $70 \%$ of diffuse grade II and III gliomas [46].

Considering that heterogeneity of TSAs in the patient population as a potentially limiting factor in treatment efficacy, tumor-associated antigens (TAAs), which are not tumor exclusive but are relatively overexpressed compared to normal tissues, may be a more viable target in tumor vaccines. Clinical trials in GBM patients, using peptide-pulsed dendritic cells or peptides alone in adjuvant, demonstrated that TAA-based vaccine could elicit T-cell responses without collateral autoimmunity, showing benefit in some patients [47-50]. Early results were exciting, prompting initiation of more clinical trials, such as applying the vaccine in patients with lower-grade glioma, oligodendroglioma, oligoastrocytoma, and ependymoma (NCT01795313). On the other hand, peptide elution from GBM cells was demonstrated capable of identifying 10 novel GBM-associated antigens, brevican, chitinase 3-like 2, Chondroitin sulphate proteoglycan, fatty acid-binding protein 7 , insulin-like growth factor 2 messenger RNA-binding protein 3, neuroligin 4, X-linked, neuronal cell adhesion molecule, protein tyrosine phosphatase, receptortype, $\mathrm{Z}$ polypeptide, tenascin $\mathrm{C}$, were overexpressed in $80-$ $100 \%$ of GBM patients, making a peptide vaccine possible [51]. In this study, researchers found $>6000$ HLA-bound peptides from HLA-A ${ }^{*} 02^{+}$glioblastoma, of which over 3000 were restricted by HLA-A* 02 . They prioritized investigation of these 10 glioblastoma-associated antigens, to which GBM patients showed no T-cell tolerance. Moreover, researchers demonstrated that these 10 peptides were highly immunogenic not only in healthy individuals but also in GBM patients, 9 of which were being developed in a multipeptide therapeutic vaccine designated IMA950. Moreover, peptide elution from GBM cells identified 10 novel GBM-associated antigens which are overexpressed in $80-100 \%$ of GBM patients, making a peptide vaccine a potential reality [51]. Three trials that incorporate these well-characterized TAAs (called the IMA950 antigens) are underway (NCT01403285, NCT01920191, NCT01222221), using CD8 ${ }^{+}$T-cell epitopes with different adjuvants. Other trials aiming at eliciting both CD4 and CD8 T-cell responses use whole proteins as immunogen to construct the TAA vaccines (NCT00626483, NCT01522820, NCT00390299).

Vaccines that target single antigens are restricted to the relatively small subset of patients with tumors that express those TSAs and TAAs. Moreover, the heterogeneity of tumor cells in expressing such antigens may also potentially limit the utility and efficacy of these single-antigen vaccines. Accordingly, alternative vaccine approaches have been created to target a broad range of antigens. Among these, heatshock protein (HSP) peptide complexes (HSPPC-96) have generated particular interest. HSPPC-96 is a primary resident chaperone of the endoplasmic reticulum and binds various client proteins that are involved in the antigen-presenting pathway [52]. When conjugating to tumor peptides, intracellular and extracellular HSPs coordinate to mediate the internalization of HSPPC-96 into APCs for efficient class I and II MHC-mediated presentation of tumor peptides [53]. Thus, HSPPC-96-tumor peptide complexes can generate potent tumor-specific immune responses. In a phase II trial for surgically resectable recurrent GBM, in which HSPPC96-loaded antigens were extracted from patient-derived glioma tissue to use as a personalized antiglioma vaccine, the median OS was increased to an impressive 42.6 weeks, a substantial survival benefit when compared to historical controls [54]. However, immunotherapeutic approaches may be complicated by immunogenic side effects profiles, for example, HSPPC-96 stimulation of both cytotoxic T lymphocytes (CTLs) and Tregs, especially at higher doses [55], and lymphopenia [54].

\section{Alternative Immune-Mediated Vaccines}

The concept of vaccine immunotherapy involves priming antigen-presenting cells (APCs) with tumor-derived antigens in order to accelerate the eradication of tumor cells [56] (Figure 1). Of the three types of professional APCs, dendritic cells (DCs) are the most powerful and efficient in activating T-cells, making DCs attractive candidates for therapeutic antitumor strategies [57]. DCs express high levels of cell surface markers MHC class I, MHC class II, and CD86 [58] and are involved in both innate and adaptive immune systems [59]. Compared to other APCs, DCs process antigens more slowly generating a longer and more sustained $\mathrm{T}$-cell response [60]. Autologous DCs exposed to GBM-associated antigens to take up and process the antigens as peptides on their cell surface in the context of MHCs are injected back into patients as a vaccine therapy. Not only can the T-cells of patients be activated by DCs-based vaccines via recognition of MHC class I or II molecule, but natural killer (NK) and natural killer T (NKT) cell function can be improved, both of which can also elicit a powerful antitumor effect [61]. The efficacy of DC-based vaccine for GBM utilizing pulsed autologous DCs with tumor lysate is currently tested in a phase III trial for newly diagnosed GBM patients (NCT00045968). A preclinical study demonstrated that modulation of CMVspecific DCs with tetanus/diphtheria (Td) preconditioning could increase DC migration to vaccine site-draining lymph nodes (VDLNs) [62]. This DC migration could also be enhanced by exogenous administration of chemokine CCL3 in a mouse model with normal CD4 T-dependent immune responses. The investigators propose CCL3 as a novel and important mediator to increase DC migration to VDLNs. In this study, researchers found that Td-treatment could 
TABLE 3: Representative clinical trials of immune checkpoint blockade in glioma.

\begin{tabular}{|c|c|c|c|c|c|}
\hline Registration number & New/recurrent/metastatic & Mechanism & Therapy & Number of patients & Phase \\
\hline NCT02017717 & Recurrent & Anti-PD1, anti-CTLA4 & $\begin{array}{c}\text { Nivolumab, ipilimumab, } \\
\text { bevacizumab }\end{array}$ & $n=440$ & Phase III \\
\hline NCT02336165 & $\mathrm{New}+$ recurrent & Anti-PDL1 & $\begin{array}{c}\text { MEDI4736, } \\
\text { Bevacizumab, }\end{array}$ & $n=84$ & Phase II \\
\hline NCT02311920 & New + recurrent & Anti-PD1, anti-CTLA4 & $\begin{array}{l}\text { TMZ, nivolumab, } \\
\text { ipilimumab }\end{array}$ & $n=42$ & Phase I \\
\hline NCT02337491 & Recurrent & Anti-PD1 & $\begin{array}{l}\text { Pembrolizumab, } \\
\text { bevacizumab }\end{array}$ & $n=79$ & Phase II \\
\hline NCT01952769 & Recurrent & Anti-PD1 & Pidilizumab & $n=30$ & Phase I/II \\
\hline
\end{tabular}

not only increase the PSF and OF in GBM patients but also suppress the tumor growth in their established mouse model. Accordingly, strategies aiming at modulating the DC migration may be a promising therapeutic option. However, the modification of autologous DCs is an expensive, timeconsuming, and labor-intensive process that must be carried out in specialized facilities. In addition, the variability of some antigens in inducing immune responses may also result in variable and inconsistent effects. Tumor-specific proteins and peptides that represent these proteins have then been used as antigens to enhance tumor-specific cytotoxicity [63].

Another approach uses an immunotherapeutic strategy to target glioma stem cells (GSCs). With their more active DNA repair mechanisms and highly expressed multidrug resistance genes, GSCs may play a role in mediating the resistance of GBM to radiotherapy and chemotherapy and contribute to local immunosuppression in the GBM microenvironment [64-66]. Several studies have demonstrated that GSC-antigens-loaded DC vaccines could induce immunereactivity and a survival benefit in rodent orthotopic GBM models $[67,68]$. Another study showed that immunization with GLAST peptides, a neural stem cell marker that is highly expressed in the plasma membrane of GSCs, could efficiently prevent the tumor progression in a glioma GL261 mouse model [69]. Clinically, a DC vaccine (ICT-107) loaded with six synthetically processed GBM-associated peptides, four of which (HER2, TRP-2, AIM-2, and IL13R $\alpha 2$ ) are considered GSC-associated, has shown promising results in phase II trial for newly diagnosed GBM patients [70]. Another phase I trial found that median PFS and OS in newly diagnosed GBM patients were 16.9 and 38.4 months, which were correlated with expression of the GSCs associated antigens in tumors before vaccination [47]. Accordingly, GSC-antigens, however, may be ideal for vaccination for their capability of stimulating T-cells to induce tumor-specific cytotoxicity against GBM cells when loaded to DCs [71].

Several studies have demonstrated that GSC-antigensloaded DC vaccines could induce immune-reactivity and a survival benefit in rodent orthotopic GBM models [69, 70]. Clinically, a DC vaccine (ICT-107) loaded with six synthetically processed GSC-associated peptides has shown promising results in phase II trials for newly diagnosed GBM patients [47]. Accordingly, GSC-antigens, however, may be ideal for vaccination for their capability of stimulating T-cells to induce tumor-specific cytotoxicity against GBM cells when loaded to DCs [71].

\section{Immune Checkpoint Inhibition}

It has been recognized that coinhibitory receptors on T-cells play an essential role in attenuating the strength and duration of T-cell-mediated immune responses. These inhibitory receptors are referred to as immune checkpoint molecules responsible for maintaining self-tolerance and preventing autoimmune reactions $[72,73]$. To date, the two most intensely investigated coinhibitory molecules are CTLA-4 (that acts early in T-cell activation) and PD-1 (that blocks Tcells at later stages of the immune response) [74]. It has been demonstrated that blockade of CTLA4 and PD1 could induce tumor regression and promote long-term survival in mouse glioma models (Table 3) [35, 75]. Clinically, ipilimumab, a humanized CTLA-4 antibody and the first FDA-approved immune checkpoint inhibitor, has been demonstrated to improve OS in a phase III clinical trial for metastatic melanoma patients [76], however, with only a complete response observed in $2 \%$ patients. In phase I and II trials of solid tumors, ipilimumab improved PFS [77, 78] but with severe immune adverse effects [79]. However, another CTLA4 antibody, tremelimumab, failed to show significant survival benefit in phase III trial for metastatic melanoma patients [74]. In GBM, robust antitumor immunity introduced by CTLA-4 mAb was only observed in at the preclinical stage [75] and the clinical utility of ipilimumab may be limited to only a small subset of GBM patients.

Conversely, efforts aimed at inhibiting the PD-1/PDL1 pathway have shown more promising results. In a preclinical study using the GL261 glioma mouse model, combination of anti-PD-1 antibodies and radiotherapy doubled median survival and elicited long-term survival in $15-40 \%$ of mice compared with either treatment alone [75]. Clinically, pembrolizumab, a PD-L1 antibody, has been approved by the FDA to apply in the treatment of metastatic melanoma and NSCLC. In GBM, nivolumab, another PD-1 antibody, developed for GBM patients is being tested, with two clinical trials currently recruiting GBM patients (NCT02337491, NCT02336165). The most promising results have been achieved in a randomized control trial with combinatorial 
CTLA-4/PD-(L)1 blockade for advanced melanoma, in which combination of CTLA- 4 and PD- 1 blockade demonstrated an improved objective response rate (ORR) of $58 \%$, compared to monotherapy of anti-CTLA-4 (19\%) and monotherapy of anti-PD-1 (44\%) [80]. A randomized phase III study aimed at testing nivolumab versus bevacizumab in recurrent GBM patients will also test combination therapy of nivolumab and ipilimumab (NCT02017717). Another two phase I/II trials will analyze the effectiveness of combinatorial pembrolizumab with bevacizumab (NCT02337491) and combinatorial pembrolizumab with MRI-guided laser ablation (NCT02311582) in recurrent GBM patients. In addition, MEDI4736, a humanized PD-Ll mAb, is currently being tested in clinical trials for GBM patients combined with radiotherapy and bevacizumab (NCT02336165).

However, relatively high frequency of immune-related adverse effects, such as endocrinological, hepatic, gastrointestinal, and dermatological toxicities, have limited enthusiasm for immune checkpoint blockade as a immunotherapeutic strategy against cancer [81]. These adverse effects were considered to be associated with aberrant infiltration of stimulated $\mathrm{CD} 4^{+}$and $\mathrm{CD} 8^{+} \mathrm{T}$-cells into normal tissues in company with elevated levels of proinflammatory cytokines [82]. Recently, newer agents targeting PD-1 ligands (PDlLs) have now been tested in renal cell cancer, NSCLC, and melanoma (NCT00729664). These agents have shown the capability of inducing durable tumor regression with less grade 3 or 4 adverse events compared with CTLA- $4 \mathrm{mAb}$ and PD-1 mAb [83]. Overall, the combination of various immune checkpoint modulators have shown promising effectiveness in the treatment of some solid tumors. The application of combinatorial checkpoint modulators in GBM and other tumors therefore requires further investigation into the interplay of costimulatory and coinhibitory molecules.

\section{Adoptive T-Cell Therapy}

While previously described therapeutic strategies endeavored to induce endogenous T-cell responses, adoptive T-cell therapies provide an alternative strategy: in vitro amplification of tumor-specific autologous T-cells followed by venous infusion into the same individual. Adoptive T-cell therapy has evolved during the past two decades in concert with the development of genetic engineering, resulting in the generation of high avidity tumor-specific T-cells. Tumorreactive T-cells are often achieved by transducing the patient's autologous T-cells with vectors encoding T-cell receptors (TCR) or chimeric antibody receptors (CAR) [84]. Although TCR engineering has not yet been applied in glioma, several preclinical studies of CARs targeting proteins (IL-13 receptor [85, 86], Her2 [87, 88], EphA2 [89], and EGFRvIII [90, 91]) have shown promising results. Clinically, adoptive T-cell therapy has demonstrated its effectiveness with CAR-based treatment for CD19C B-cell malignancies [92]. A clinical trial for 11 recurrent GBM patients has demonstrated infusions of autologous adoptively transferred human cytomegalovirus(CMV-) specific T-cells increased OS to of $>57$ weeks, with 4 patients maintaining no progression throughout the study period [93]. Another clinical trial concerning CMV adoptive T-cell therapy is ongoing (NCT00693095). The next step of adoptive T-cell therapy for GBM patients will likely involve transducing autologous T-cells with CAR. CAR, which consist of the antigen-binding region of a monoclonal antibody fused with a T-cell cytoplasmic signaling domain, acts independently of MHC I expression on tumors [94]. Clinical trials investigating CAR targeting EGFRvIII (NCT02209376, NCT01454596), HER2 (NCT01109095), and IL-13R $\alpha 2$ (NCT02208362) are underway, and therapeutic benefits without unacceptable toxicity are anticipated.

\section{Conclusions}

Current open clinical trials of immunotherapy predominantly focusing on DC vaccines and antibodies targeting immunosuppressive checkpoints have achieved promising immune activity and clinical responses (see Tables 1 and 2 for summary). However, durable and sustained responses remain rare, highlighting the need for novel promising approaches including gene therapy and combinatorial immunotherapeutic treatment. Immunogenic side effect profiles underlie the need for next-generation immunotherapies with nonimmunosuppressive and/or anti-inflammatory approaches. Current obstacles for immune therapy for GBM lie in (1) finding drugs to penetrate the BBB; (2) identifying specific, suitable, and immunogenic tumor antigens; and (3) identifying appropriate pre- and posttherapeutic biomarkers. Other challenges include the limited number of GBM patients eligible to join particular clinical studies and a deep understanding of various regulatory and stimulatory factors in the immune system and GBM microenvironment. Considering that the brain tumors will ultimately metastasize outside the CNS, one future direction of immunotherapy is to design immunotherapies to obtain sufficient functional antitumor T-cell in the CNS, with no other sites to be targeted. If so, one challenge will be determining tolerable levels of inflammation to occur without damage to the brain. Additionally, there remains a need for standardized and validated assays to measure the immune response. However, increased efforts have been dedicated to establishing reliable biomarkers to improve the assessment of clinical efficacy to guide therapeutic decision-making [95]. Immune therapy for GBM requires an integrated effort, with combinations of vaccines, cell therapy, and molecules targeting the tumor environment, trying as well to exploit the beneficial aspects of radio- and chemotherapy. This will serve to improve and promote the development of an optimal personalized therapeutic strategy for the treatment of GBM.

\section{Competing Interests}

The authors have no conflict of interests.

\section{Authors' Contributions}

Boyuan Huang and Hongbo Zhang equally contributed to this work. 


\section{Acknowledgments}

This work was supported by the National Natural Science Foundation of China (no. 81571147 to Xiaoxing Xiong, no. 81301019 to Lijuan Gu, and no. 81600101 to Bainxin Ye) and an American Heart Association Award 14FTF19970029 to Creed Stary, and the Fundamental Research Funds for the Central Universities (no. 2042016kf0078 to Bainxin Ye).

\section{References}

[1] P. Y. Wen and S. Kesari, "Malignant gliomas in adults," New England Journal of Medicine, vol. 359, no. 5, pp. 492-507, 2008.

[2] Q. T. Ostrom, H. Gittleman, P. Liao et al., "CBTRUS statistical report: primary brain and central nervous system tumors diagnosed in the United States in 2007-2011," Neuro-oncology, vol. 16, pp. iv1-iv63, 2014.

[3] M. Weller, M. van den Bent, K. Hopkins et al., "EANO guideline for the diagnosis and treatment of anaplastic gliomas and glioblastoma," The Lancet Oncology, vol. 15, no. 9, pp. e395-e403, 2014.

[4] R. Stupp, W. P. Mason, M. J. Van Den Bent et al., "Radiotherapy plus concomitant and adjuvant temozolomide for glioblastoma," New England Journal of Medicine, vol. 352, no. 10, pp. 987-996, 2005.

[5] S. J. O’Day, M. Maio, V. Chiarion-Sileni et al., "Efficacy and safety of ipilimumab monotherapy in patients with pretreated advanced melanoma: A Multicenter Single-arm Phase Ii Study," Annals of Oncology, vol. 21, no. 8, pp. 1712-1717, 2010.

[6] J. D. Wolchok, H. Kluger, M. K. Callahan et al., "Nivolumab plus Ipilimumab in advanced melanoma," New England Journal of Medicine, vol. 369, no. 2, pp. 122-133, 2013.

[7] S. M. Ansell, A. M. Lesokhin, I. Borrello et al., "PD-1 blockade with nivolumab in relapsed or refractory Hodgkin's lymphoma," The New England Journal of Medicine, vol. 372, no. 4, pp. 311-319, 2015.

[8] N. A. Rizvi, J. Mazières, D. Planchard et al., "Activity and safety of nivolumab, an anti-PD-1 immune checkpoint inhibitor, for patients with advanced, refractory squamous non-small-cell lung cancer (CheckMate 063): a phase 2, single-arm trial," The Lancet Oncology, vol. 16, no. 3, pp. 257-265, 2015.

[9] R. J. Motzer, B. I. Rini, D. F. McDermott et al., "Nivolumab for metastatic renal cell carcinoma: results of a randomized phase II trial," Journal of Clinical Oncology, vol. 33, no. 13, pp. 1430-1437, 2015.

[10] C. Jackson, J. Ruzevick, J. Phallen, Z. Belcaid, and M. Lim, "Challenges in immunotherapy presented by the glioblastoma multiforme microenvironment," Clinical and Developmental Immunology, vol. 2011, Article ID 732413, 20 pages, 2011.

[11] A. B. Heimberger and J. H. Sampson, "Immunotherapy coming of age: what will it take to make it standard of care for glioblastoma?" Neuro-Oncology, vol. 13, no. 1, pp. 3-13, 2011.

[12] M. J. Carson, J. M. Doose, B. Melchior, C. D. Schmid, and C. C. Ploix, "CNS immune privilege: hiding in plain sight," Immunological Reviews, vol. 213, no. 1, pp. 48-65, 2006.

[13] E. Vauleon, T. Avril, B. Collet, J. Mosser, and V. Quillien, "Overview of cellular immunotherapy for patients with glioblastoma," Clinical and Developmental Immunology, vol. 2010, Article ID 689171, 18 pages, 2010.

[14] T. Calzascia, F. Masson, W. Di Berardino-Besson et al., "Homing phenotypes of tumor-specific CD8 T cells are predetermined at the tumor site by crosspresenting APCs," Immunity, vol. 22, no. 2, pp. 175-184, 2005.

[15] F. Masson, T. Calzascia, W. Di Berardino-Besson, N. De Tribolet, P.-Y. Dietrich, and P. R. Walker, "Brain microenvironment promotes the final functional maturation of tumor-specific effector CD8+ T cells," Journal of Immunology, vol. 179, no. 2, pp. 845-853, 2007.

[16] J. M. Pitt, A. Marabelle, A. Eggermont, J. Soria, G. Kroemer, and L. Zitvogel, "Targeting the tumor microenvironment: removing obstruction to anticancer immune responses and immunotherapy," Annals of Oncology, vol. 27, no. 8, pp. 1482-1492, 2016.

[17] S. Lively and L. C. Schlichter, "The microglial activation state regulates migration and roles of matrix-dissolving enzymes for invasion," Journal of Neuroinflammation, vol. 10, article no. 75, 2013.

[18] F. Szulzewsky, S. Arora, L. de Witte et al., "Human glioblastomaassociated microglia/monocytes express a distinct RNA profile compared to human control and murine samples," GLIA, vol. 64, no. 8, pp. 1416-1436, 2016.

[19] S. Rivest, "Regulation of innate immune responses in the brain," Nature Reviews Immunology, vol. 9, no. 6, pp. 429-439, 2009.

[20] A. Farin, S. O. Suzuki, M. Weiker, J. E. Goldman, J. N. Bruce, and P. Canoll, "Transplanted glioma cells migrate and proliferate on host brain vasculature: a dynamic analysis," Glia, vol. 53, no. 8 , pp. 799-808, 2006.

[21] S. Watkins, S. Robel, I. F. Kimbrough, S. M. Robert, G. EllisDavies, and H. Sontheimer, "Disruption of astrocyte-vascular coupling and the blood-brain barrier by invading glioma cells," Nature Communications, vol. 5, article no. 4196, 2014.

[22] T. T. Tran, M. UhI, J. Y. Ma et al., "Inhibiting TGF- $\beta$ signaling restores immune surveillance in the SMA-560 glioma model," Neuro-Oncology, vol. 9, no. 3, pp. 259-270, 2007.

[23] D. A. Wainwright, A. L. Chang, M. Dey et al., "Durable therapeutic efficacy utilizing combinatorial blockade against IDO, CTLA-4, and PD-L1 in mice with brain tumors," Clinical Cancer Research, vol. 20, no. 20, pp. 5290-5301, 2014.

[24] A. T. Parsa, J. S. Waldron, A. Panner et al., "Loss of tumor suppressor PTEN function increases B7-H1 expression and immunoresistance in glioma," Nature Medicine, vol. 13, no. 1, pp. 84-88, 2007.

[25] K. Mitsuka, T. Kawataki, E. Satoh, T. Asahara, T. Horikoshi, and H. Kinouchi, "Expression of indoleamine 2,3-dioxygenase and correlation with pathological malignancy in gliomas," Neurosurgery, vol. 72, no. 6, pp. 1031-1038, 2013.

[26] S. Herbertz, J. S. Sawyer, A. J. Stauber et al., "Clinical development of galunisertib (Ly2157299 monohydrate), a small molecule inhibitor of transforming growth factor-beta signaling pathway," Drug Design, Development and Therapy, vol. 9, pp. 4479-4499, 2015.

[27] L. Zhai, K. L. Lauing, A. L. Chang et al., "The role of IDO in brain tumor immunotherapy," Journal of Neuro-Oncology, vol. 123, no. 3, pp. 395-403, 2015.

[28] A. S. Berghoff, B. Kiesel, G. Widhalm et al., "Programmed death ligand 1 expression and tumor-infiltrating lymphocytes in glioblastoma," Neuro-Oncology, vol. 17, no. 8, pp. 1064-1075, 2015.

[29] J. S. Weber, R. R. Kudchadkar, B. Yu et al., "Safety, efficacy, and biomarkers of nivolumab with vaccine in ipilimumabrefractory or -naive melanoma," Journal of Clinical Oncology, vol. 31, no. 34, pp. 4311-4318, 2013. 
[30] V. Velcheti, K. A. Schalper, D. E. Carvajal et al., "Programmed death ligand-1 expression in non-small cell lung cancer," Laboratory Investigation, vol. 94, no. 1, pp. 107-116, 2014.

[31] O. Bloch, C. A. Crane, R. Kaur, M. Safaee, M. J. Rutkowski, and A. T. Parsa, "Gliomas promote immunosuppression through induction of B7-H1 expression in tumor-associated macrophages," Clinical Cancer Research, vol. 19, no. 12, pp. 31653175, 2013.

[32] A. S. Berghoff, B. Kiesel, G. Widhalm et al., "Programmed death ligand 1 expression and tumor-infiltrating lymphocytes in glioblastoma," Neuro-Oncology, vol. 17, no. 8, pp. 1064-1075, 2014.

[33] M. F. Krummel and J. P. Allison, "CD28 and CTLA-4 have opposing effects on the response of T cells to stimulation," Journal of Experimental Medicine, vol. 182, no. 2, pp. 459-465, 1995.

[34] P. S. Linsley, W. Brady, M. Urnes, L. S. Grosmaire, N. K. Damle, and J. A. Ledbetter, "CTLA-4 is a second receptor for the B cell activation antigen B7," The Journal of Experimental Medicine, vol. 174, no. 3, pp. 561-569, 1991.

[35] P. E. Fecci, H. Ochiai, D. A. Mitchell et al., "Systemic CTLA-4 blockade ameliorates glioma-induced changes to the $\mathrm{CD} 4^{+} \mathrm{T}$ cell compartment without affecting regulatory T-cell function," Clinical Cancer Research, vol. 13, no. 7, pp. 2158-2167, 2007.

[36] I. Mellman, G. Coukos, and G. Dranoff, "Cancer immunotherapy comes of age," Nature, vol. 480, no. 7378, pp. 480-489, 2011.

[37] P. C. Gedeon, B. D. Choi, J. H. Sampson, and D. D. Bigner, "Rindopepimut: anti-EGFRvIII peptide vaccine oncolytic," Drugs of the Future, vol. 38, no. 3, pp. 147-155, 2013.

[38] J. H. Sampson, A. B. Heimberger, G. E. Archer et al., "Immunologic escape after prolonged progression-free survival with epidermal growth factor receptor variant III peptide vaccination in patients with newly diagnosed glioblastoma," Journal of Clinical Oncology, vol. 28, no. 31, pp. 4722-4729, 2010.

[39] J. H. Sampson, K. D. Aldape, G. E. Archer et al., "Greater chemotherapy-induced lymphopenia enhances tumor-specific immune responses that eliminate EGFRvIII-expressing tumor cells in patients with glioblastoma," Neuro-Oncology, vol. 13, no. 3, pp. 324-333, 2011.

[40] J. Schuster, R. K. Lai, L. D. Recht et al., "A phase II, multicenter trial of rindopepimut (CDX-110) in newly diagnosed glioblastoma: the ACT III study," Neuro-Oncology, vol. 17, no. 6, pp. 854861, 2015.

[41] A. Marucci, Rindopepimut Misses OS Endpoint in Phase III Glioblastoma Trial, OncLive, Cranbury, NJ, USA, 2016, http://www .abta.org/about-us/news/brain-tumor-news/rindopepimut-missesOS-endpoint.html.

[42] C. A. Del Vecchio, C. P. Giacomini, H. Vogel et al., "EGFRvIII gene rearrangement is an early event in glioblastoma tumorigenesis and expression defines a hierarchy modulated by epigenetic mechanisms," Oncogene, vol. 32, no. 21, pp. 2670-2681, 2013.

[43] J. M. Francis, C.-Z. Zhang, C. L. Maire et al., "EGFR variant heterogeneity in glioblastoma resolved through single-nucleus sequencing," Cancer Discovery, vol. 4, no. 8, pp. 956-971, 2014.

[44] C. E. Pelloski, K. V. Ballman, A. F. Furth et al., "Epidermal growth factor receptor variant III status defines clinically distinct subtypes of glioblastoma," Journal of Clinical Oncology, vol. 25, no. 16, pp. 2288-2294, 2007.

[45] T. Schumacher, L. Bunse, S. Pusch et al., "A vaccine targeting mutant IDH1 induces antitumour immunity," Nature, vol. 512, no. 7514, pp. 324-327, 2014.
[46] H. Yan, D. W. Parsons, G. Jin et al., "IDH1 and IDH2 mutations in gliomas," The New England Journal of Medicine, vol. 360, no. 8, pp. 765-773, 2009.

[47] S. Phuphanich, C. J. Wheeler, J. D. Rudnick et al., "Phase I trial of a multi-epitope-pulsed dendritic cell vaccine for patients with newly diagnosed glioblastoma," Cancer Immunology, Immunotherapy, vol. 62, no. 1, pp. 125-135, 2013.

[48] H. Okada, P. Kalinski, R. Ueda et al., "Induction of $\mathrm{CD}^{+}$Tcell responses against novel glioma-associated antigen peptides and clinical activity by vaccinations with $\alpha$-type 1 polarized dendritic cells and polyinosinic-polycytidylic acid stabilized by lysine and carboxymethylcellulose in patients with recurrent malignant glioma," Journal of Clinical Oncology, vol. 29, no. 3, pp. 330-336, 2011.

[49] M. Terasaki, S. Shibui, Y. Narita et al., "Phase I trial of a personalized peptide vaccine for patients positive for human leukocyte antigen-A24 with recurrent or progressive glioblastoma multiforme," Journal of Clinical Oncology, vol. 29, no. 3, pp. 337-344, 2011.

[50] I. F. Pollack, R. I. Jakacki, L. H. Butterfield et al., "Antigenspecific immune responses and clinical outcome after vaccination with glioma-associated antigen peptides and polyinosinicpolycytidylic acid stabilized by lysine and carboxymethylcellulose in children with newly diagnosed malignant brainstem and nonbrainstem gliomas," Journal of Clinical Oncology, vol. 32, no. 19, pp. 2050-2058, 2014.

[51] V. Dutoit, C. Herold-Mende, N. Hilf et al., "Exploiting the glioblastoma peptidome to discover novel tumour-associated antigens for immunotherapy," Brain, vol. 135, no. 4, pp. 10421054, 2012.

[52] R. J. Amato, "Heat-shock protein-peptide complex-96 for the treatment of cancer," Expert Opinion on Biological Therapy, vol. 7, no. 8, pp. 1267-1273, 2007.

[53] S. Basu, R. J. Binder, T. Ramalingam, and P. K. Srivastava, "CD91 is a common receptor for heat shock proteins gp96, hsp90, hsp70, and calreticulin," Immunity, vol. 14, no. 3, pp. 303-313, 2001.

[54] O. Bloch, C. A. Crane, Y. Fuks et al., "Heat-shock protein peptide complex-96 vaccination for recurrent glioblastoma: a phase II, single-arm trial," Neuro-Oncology, vol. 16, no. 2, pp. 274-279, 2014.

[55] Z. Liu, X. Li, L. Qiu et al., "Treg suppress CTL responses upon immunization with HSP gp96," European Journal of Immunology, vol. 39, no. 11, pp. 3110-3120, 2009.

[56] A. Bregy, T. M. Wong, A. H. Shah, J. M. Goldberg, and R. J. Komotar, "Active immunotherapy using dendritic cells in the treatment of glioblastoma multiforme," Cancer Treatment Reviews, vol. 39, no. 8, pp. 891-907, 2013.

[57] K. Palucka and J. Banchereau, "Cancer immunotherapy via dendritic cells," Nature Reviews Cancer, vol. 12, no. 4, pp. 265277,2012

[58] L. H. Butterfield, "Dendritic cells in cancer immunotherapy clinical trials: are we making progress?" Frontiers in Immunology, vol. 4, article no. 454, 2013.

[59] X. Jie, L. Hua, W. Jiang, F. Feng, G. Feng, and Z. Hua, “Clinical application of a dendritic cell vaccine raised against heatshocked glioblastoma," Cell Biochemistry and Biophysics, vol. 62, no. 1, pp. 91-99, 2012.

[60] C. Goyvaerts and K. Breckpot, "Pros and cons of antigenpresenting cell targeted tumor vaccines," Journal of Immunology Research, vol. 2015, Article ID 785634, 18 pages, 2015. 
[61] M.-Y. Yang, P. M. Zetler, R. M. Prins, H. Khan-Farooqi, and L. M. Liau, "Immunotherapy for patients with malignant glioma: from theoretical principles to clinical applications," Expert Review of Neurotherapeutics, vol. 6, no. 10, pp. 1481-1494, 2006.

[62] D. A. Mitchell, K. A. Batich, M. D. Gunn et al., "Tetanus toxoid and CCL3 improve dendritic cell vaccines in mice and glioblastoma patients," Nature, vol. 519, no. 7543, pp. 366-369, 2015.

[63] J. A. Berzofsky, M. Terabe, S. Oh et al., "Progress on new vaccine strategies for the immunotherapy and prevention of cancer," Journal of Clinical Investigation, vol. 113, no. 11, pp. 1515-1525, 2004.

[64] S. Bao, Q. Wu, R. E. McLendon et al., "Glioma stem cells promote radioresistance by preferential activation of the DNA damage response," Nature, vol. 444, no. 7120, pp. 756-760, 2006.

[65] A.-M. Bleau, D. Hambardzumyan, T. Ozawa et al., "PTEN/ $\mathrm{PI} 3 \mathrm{~K} / \mathrm{Akt}$ pathway regulates the side population phenotype and ABCG2 activity in glioma tumor stem-like cells," Cell Stem Cell, vol. 4, no. 3, pp. 226-235, 2009.

[66] A. Wu, J. Wei, L.-Y. Kong et al., "Glioma cancer stem cells induce immunosuppressive macrophages/microglia," Neuro-Oncology, vol. 12, no. 11, pp. 1113-1125, 2010.

[67] S. Pellegatta, P. L. Poliani, D. Corno et al., "Neurospheres enriched in cancer stem-like cells are highly effective in eliciting a dendritic cell-mediated immune response against malignant gliomas," Cancer Research, vol. 66, no. 21, pp. 10247-10252, 2006.

[68] Q. Xu, G. Liu, X. Yuan et al., "Antigen-specific T-cell response from dendritic cell vaccination using cancer stem-like cellassociated antigens," Stem Cells, vol. 27, no. 8, pp. 1734-1740, 2009.

[69] G. Cantini, F. Pisati, S. Pessina, G. Finocchiaro, and S. Pellegatta, "Immunotherapy against the radial glia marker GLAST effectively triggers specific antitumor effectors without autoimmunity," OncoImmunology, vol. 1, no. 6, pp. 884-893, 2012.

[70] P. Wen, D. Reardon, S. Phuphanich et al., "AT-60-a randomized double blind placebo-controlled phase 2 trial of dendritic cell (DC) vaccine ICT-107 following standard treatment in newly diagnosed patients with GBM," Neuro-Oncology, vol. 16, no. 5, article v22, 2014.

[71] B. Ji, Q. Chen, B. Liu et al., "Glioma stem cell-targeted dendritic cells as a tumor vaccine against malignant glioma," Yonsei Medical Journal, vol. 54, no. 1, pp. 92-100, 2013.

[72] B. Engels, V. H. Engelhard, J. Sidney et al., "Relapse or eradication of cancer is predicted by peptide-major histocompatibility complex affinity," Cancer Cell, vol. 23, no. 4, pp. 516-526, 2013.

[73] P. F. Robbins, Y.-C. Lu, M. El-Gamil et al., "Mining exomic sequencing data to identify mutated antigens recognized by adoptively transferred tumor-reactive T cells," Nature Medicine, vol. 19, no. 6, pp. 747-752, 2013.

[74] D. M. Pardoll, "The blockade of immune checkpoints in cancer immunotherapy," Nature Reviews Cancer, vol. 12, no. 4, pp. 252264, 2012.

[75] J. Zeng, A. P. See, J. Phallen et al., "Anti-PD-1 blockade and stereotactic radiation produce long-term survival in mice with intracranial gliomas," International Journal of Radiation Oncology Biology Physics, vol. 86, no. 2, pp. 343-349, 2013.

[76] F. S. Hodi, S. J. O’Day, D. F. McDermott et al., "Improved survival with ipilimumab in patients with metastatic melanoma," The New England Journal of Medicine, vol. 363, no. 8, pp. 711723, 2010 .
[77] T. J. Lynch, I. Bondarenko, A. Luft et al., "Ipilimumab in combination with paclitaxel and carboplatin as first-line treatment in stage IIIB/IV non-small-cell lung cancer: results from a randomized, double-blind, multicenter phase II study," Journal of Clinical Oncology, vol. 30, no. 17, pp. 2046-2054, 2012.

[78] J. C. Yang, M. Hughes, U. Kammula et al., "Ipilimumab (antiCTLA4 antibody) causes regression of metastatic renal cell cancer associated with enteritis and hypophysitis," Journal of Immunotherapy, vol. 30, no. 8, pp. 825-830, 2007.

[79] A. Ribas, R. Kefford, M. A. Marshall et al., "Phase III randomized clinical trial comparing tremelimumab with standardof-care chemotherapy in patients with advanced melanoma," Journal of Clinical Oncology, vol. 31, no. 5, pp. 616-622, 2013.

[80] J. Larkin, V. Chiarion-Sileni, R. Gonzalez et al., "Combined nivolumab and ipilimumab or monotherapy in untreated Melanoma," New England Journal of Medicine, vol. 373, no. 1, pp. 23-34, 2015.

[81] L. Gelao, C. Criscitiello, A. Esposito, A. Goldhirsch, and G. Curigliano, "Immune checkpoint blockade in cancer treatment: a double-edged sword cross-targeting the host as an 'innocent bystander"' Toxins, vol. 6, no. 3, pp. 914-933, 2014.

[82] K. C. Kaehler, S. Piel, E. Livingstone, B. Schilling, A. Hauschild, and D. Schadendorf, "Update on immunologic therapy with anti-CTLA-4 antibodies in melanoma: identification of clinical and biological response patterns, immune-related adverse events, and their management," Seminars in Oncology, vol. 37, no. 5, pp. 485-498, 2010.

[83] J. R. Brahmer, S. S. Tykodi, L. Q. M. Chow et al., "Safety and activity of anti-PD-L1 antibody in patients with advanced cancer," New England Journal of Medicine, vol. 366, no. 26, pp. 2455-2465, 2012.

[84] S. A. Rosenberg and N. P. Restifo, "Adoptive cell transfer as personalized immunotherapy for human cancer," Science, vol. 348, no. 6230, pp. 62-68, 2015.

[85] C. E. Brown, R. Starr, B. Aguilar et al., "Stem-like tumorinitiating cells isolated from IL13R $\alpha 2$ expressing gliomas are targeted and killed by IL13-zetakine-redirected T cells," Clinical Cancer Research, vol. 18, no. 8, pp. 2199-2209, 2012.

[86] S. Kong, S. Sengupta, B. Tyler et al., "Suppression of human glioma xenografts with second-generation IL13R-specific chimeric antigen receptor-modified T cells," Clinical Cancer Research, vol. 18, no. 21, pp. 5949-5960, 2012.

[87] M. Hegde, A. Corder, K. K. Chow et al., "Combinational Targeting offsets antigen escape and enhances effector functions of adoptively transferred $\mathrm{T}$ cells in glioblastoma," Molecular Therapy, vol. 21, no. 11, pp. 2087-2101, 2013.

[88] N. Ahmed, V. S. Salsman, Y. Kew et al., "HER2-specific T cells target primary glioblastoma stem cells and induce regression of autologous experimental tumors," Clinical Cancer Research, vol. 16, no. 2, pp. 474-485, 2010.

[89] K. K. Chow, S. Naik, S. Kakarla et al., "T cells redirected to EphA2 for the immunotherapy of glioblastoma," Molecular Therapy, vol. 21, no. 3, pp. 629-637, 2013.

[90] M. Ohno, T. Ohkuri, A. Kosaka et al., "Expression of miR17-92 enhances anti-tumor activity of T-cells transduced with the anti-EGFRvIII chimeric antigen receptor in mice bearing human GBM xenografts," Journal for Immuno Therapy of Cancer, vol. 1, no. 1, p. 21, 2013.

[91] J. H. Sampson, B. D. Choi, L. Sanchez-Perez et al., "EGFRvIII mCAR-modified T-cell therapy cures mice with established intracerebral glioma and generates host immunity against 
tumor-antigen loss," Clinical Cancer Research, vol. 20, no. 4, pp. 972-984, 2014.

[92] S. L. Maude, N. Frey, P. A. Shaw et al., "Chimeric antigen receptor T cells for sustained remissions in leukemia," New England Journal of Medicine, vol. 371, no. 16, pp. 1507-1517, 2014.

[93] A. Schuessler, C. Smith, L. Beagley et al., "Autologous t-cell therapy for cytomegalovirus as a consolidative treatment for recurrent glioblastoma," Cancer Research, vol. 74, no. 13, pp. 3466-3476, 2014.

[94] H. Ikeda and H. Shiku, "Antigen-receptor gene-modified T cells for treatment of glioma," Advances in Experimental Medicine and Biology, vol. 746, pp. 202-215, 2012.

[95] J. S. Sims, T. H. Ung, J. A. Neira, P. Canoll, and J. N. Bruce, "Biomarkers for glioma immunotherapy: the next generation," Journal of Neuro-Oncology, vol. 123, no. 3, pp. 359-372, 2015.

[96] J. H. Sampson, G. E. Archer, D. A. Mitchell et al., "An epidermal growth factor receptor variant III-targeted vaccine is safe and immunogenic in patients with glioblastoma multiforme," Molecular Cancer Therapeutics, vol. 8, no. 10, pp. 2773-2779, 2009.

[97] M. A. Shevtsov, A. V. Kim, K. A. Samochernych et al., "Pilot study of intratumoral injection of recombinant heat shock protein 70 in the treatment of malignant brain tumors in children," OncoTargets and Therapy, vol. 7, pp. 1071-1081, 2014.

[98] C. E. Fadul, J. L. Fisher, T. H. Hampton et al., "Immune response in patients with newly diagnosed glioblastoma multiforme treated with intranodal autologous tumor lysatedendritic cell vaccination after radiation chemotherapy," Journal of Immunotherapy, vol. 34, no. 4, pp. 382-389, 2011.

[99] H. Ardon, S. Van Gool, I. S. Lopes et al., "Integration of autologous dendritic cell-based immunotherapy in the primary treatment for patients with newly diagnosed glioblastoma multiforme: a pilot study," Journal of Neuro-Oncology, vol. 99, no. 2, pp. 261-272, 2010.

[100] R. D. Valle, "Dendritic cell vaccination in glioblastoma after fluorescence-guided resection," World Journal of Clinical Oncology, vol. 3, no. 11, pp. 142-149, 2012.

[101] Y. Akiyama, C. Oshita, A. Kume et al., " $\alpha$-type-1 polarized dendritic cell-based vaccination in recurrent high-grade glioma: a phase I clinical trial," BMC Cancer, vol. 12, article no. 623, 2012.

[102] C.-N. Chang, Y.-C. Huang, D.-M. Yang et al., "A phase I/II clinical trial investigating the adverse and therapeutic effects of a postoperative autologous dendritic cell tumor vaccine in patients with malignant glioma," Journal of Clinical Neuroscience, vol. 18, no. 8, pp. 1048-1054, 2011. 


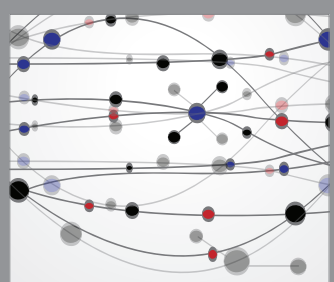

The Scientific World Journal
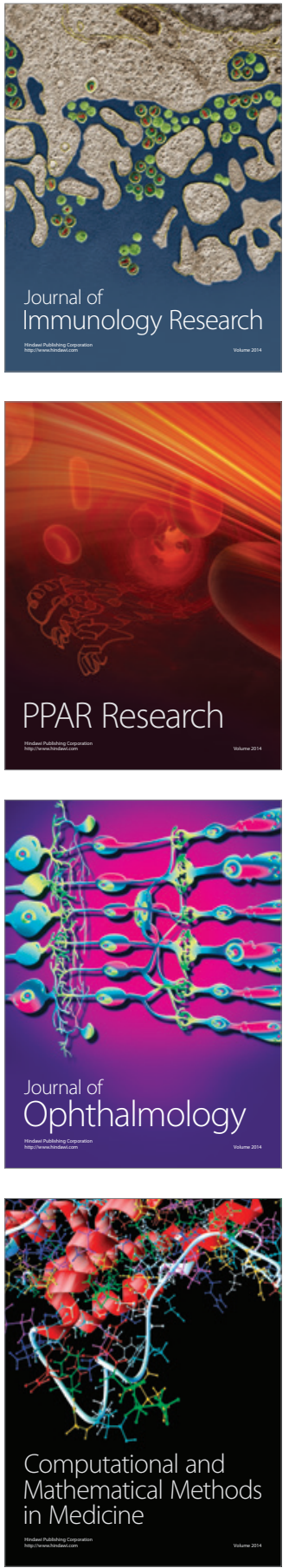

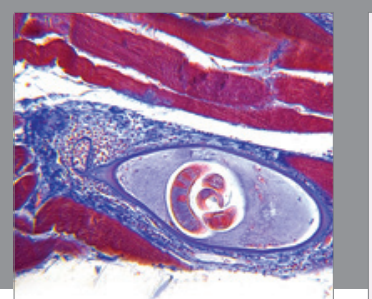

Gastroenterology Research and Practice
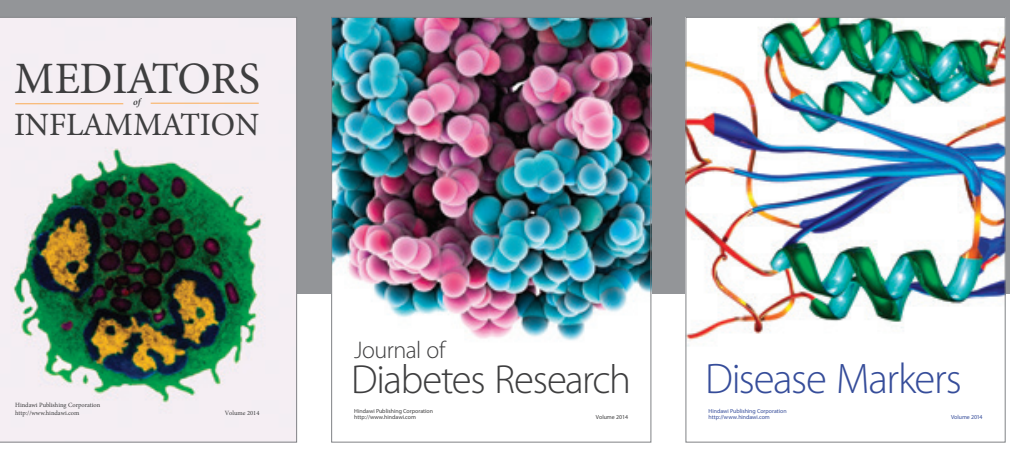

Disease Markers

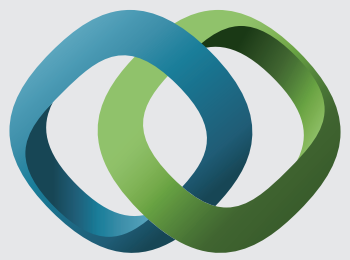

\section{Hindawi}

Submit your manuscripts at

https://www.hindawi.com
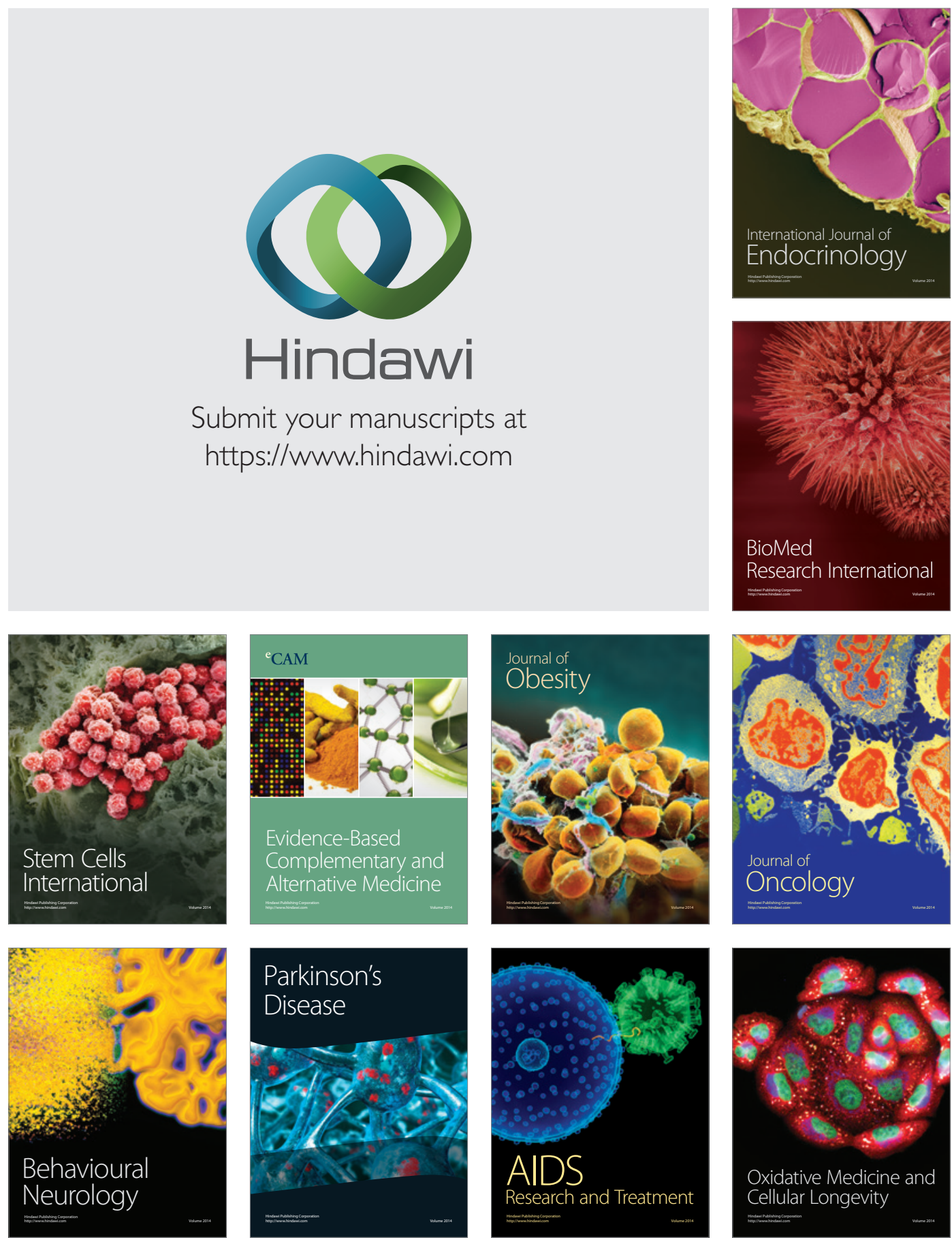